\title{
Dirac Quasinormal frequencies of the Kerr-Newman black hole
}

\author{
Jiliang Jing* and Qiyuan Pan \\ Institute of Physics and Department of Physics, \\ Hunan Normal University, \\ Changsha, Hunan 410081, P. R. China
}

\begin{abstract}
The Dirac quasinormal modes (QNMs) of the Kerr-Newman black hole are investigated using continued fraction approach. It is shown that the quasinormal frequencies in the complex $\omega$ plane move counterclockwise as the charge or angular momentum per unit mass of the black hole increases. They get a spiral-like shape, moving out of their Schwarzschild or Reissner-Nordström values and "looping in" towards some limiting frequencies as the charge and angular momentum per unit mass tend to their extremal values. The number of the spirals increases as the overtone number increases but decreases as the angular quantum number increases. It is also found that both the real and imaginary parts are oscillatory functions of the angular momentum per unit mass, and the oscillation becomes faster as the overtone number increases but slower as the angular quantum number increases.
\end{abstract}

PACS numbers: 04.70.Dy, 04.30.Db, 04.70.Bw, 97.60.Lf

*Electronic address: jljing@hunnu.edu.cn 


\section{INTRODUCTION}

It is well known that QNMs possess discrete spectra of complex characteristic frequencies which are entirely fixed by the structure of the background spacetime and irrelevant of the initial perturbations [1] [2]. Thus, it is believed that one can directly identify the existence of a black hole by comparing QNMs with the gravitational waves observed in the universe, as well as test the stability of the event horizon against small perturbations. Meanwhile, it is generally believed that the study of QNMs may lead to a deeper understanding of the thermodynamic properties of black holes in loop quantum gravity [3] 4] since the real part of quasinormal frequencies with a large imaginary part for the scalar field in the Schwarzschild black hole is equal to the Barbero-Immirzi parameter [3, 4, 5, 6], a factor introduced by hand in order that the loop quantum gravity reproduces correct entropy of the black hole. It was also argued that the QNMs of Anti-de Sitter (AdS) black holes have a direct interpretation in terms of the dual conformal field theory (CFT) [7, 8, 9]. According to the AdS/CFT correspondence, a large static black hole in asymptotically AdS spacetime corresponds to a (approximately) thermal state in CFT, and the decay of the test field in the black-hole spacetime corresponds to the decay of the perturbed state in CFT. The dynamical timescale for the return to the thermal equilibrium can be done in the AdS spacetime, and then translated into the CFT using the AdS/CFT correspondence. Thus, QNMs for various black holes have been studied extensively [10]-[29].

Although many authors studied the QNMs of scalar, electromagnetic and gravitational perturbations, the investigation of the Dirac QNMs is very limited [30, 31, 32, 33, 34]. Furthermore, in these papers the study of the Dirac QNMs was limited to use Pöshl-Teller potential or WKB approximative methods. The Pöshl-Teller potential approximative may be a valuable tool in certain situations, but in general results obtained from the approximating potentials should not be expected to be accurate. The WKB method can be generalized to deal with the Reissner-Nordström and Kerr black holes. In the latter case, unfortunately, the method is not very simple to apply and does not yield very accurate results. It is well known that the Leaver's continued fraction technique is the best workhorse method to compute highly damped modes and is very reliable. However, the method can not be directly used to study the QNMs of the Dirac fields if we take the standard wave equations. The reason to obstruct the study of the Dirac QNMs using continued fraction technique is that for static black holes the standard wave equation

$$
\left(\frac{d^{2}}{d r_{*}^{2}}+\omega^{2}\right) Z_{ \pm}=V_{ \pm} Z_{ \pm}
$$

possesses a special potential

$$
V_{ \pm}=\lambda^{2} \frac{\Delta}{r^{4}} \pm \lambda \frac{d}{d r_{*}} \frac{\sqrt{\Delta}}{r^{2}}
$$


which is the function of $\sqrt{\Delta}$ [30] 34] 35], where $\Delta$ is a function related to the metric of the spacetimes, say $\Delta=r^{2}-2 M r$ for the Schwarzschild black hole. We can not investigate the Dirac QNMs using the continued fraction approaches because we have to expand the potential as a series at the event horizon but the factor $\sqrt{\Delta}$ does not permit us to do that.

Recently, we [36, 37] found that the wave function and the potential of the Dirac fields can be expressed as new forms. Starting from the new wave function and potential, we 36, 37] studied the Dirac QNMs of the Schwarzschild black hole using the continued fraction 38] and Hill-determinant approaches [39] 40] and the Dirac QNMs of the Schwarzschild-anti-de Sitter and Reissner-Nordström-anti-de Sitter black holes using Horowitz-Hubeny approach [11]. The main purpose of this paper is to extend the investigation to the Kerr-Newman black hole because this black hole is the only asymptotically flat electro-vacuum solution of the EinsteinMaxwell system [41] and it is the most general black hole among classical black holes.

The organization of this paper is as follows. In Sec. 2 the decoupled Dirac equations and corresponding wave equations in the Kerr-Newman spacetime are obtained using NewmanPenrose formalism. In Sec. 3 the angular and radial continued fraction equations are introduced. In Sec. 4 the numerical results for the Dirac QNMs in the Reissner-Nordström, Kerr and Kerr-Newman black holes are presented. The last section is devoted to a summary.

\section{DIRAC EQUATIONS IN THE KERR-NEWMAN SPACETIME}

In a curve spacetime the Dirac equations [42] can be expressed as

$$
\begin{aligned}
& \sqrt{2} \nabla_{B B^{\prime}} P^{B}+i \mu \bar{Q}_{B^{\prime}}=0, \\
& \sqrt{2} \nabla_{B B^{\prime}} Q^{B}+i \mu \bar{P}_{B^{\prime}}=0,
\end{aligned}
$$

where $\nabla_{B B^{\prime}}$ is covariant differentiation, $P^{B}$ and $Q^{B}$ are the two-component spinors representing the wave functions, $\bar{P}_{B^{\prime}}$ is the complex conjugate of $P_{B}$, and $\mu$ is the mass of the Dirac particle. In the Newman-Penrose formalism [43] the equations become

$$
\begin{aligned}
& (D+\epsilon-\rho) P^{0}+(\bar{\delta}+\pi-\alpha) P^{1}=2^{-1 / 2} i \mu \bar{Q}^{1^{\prime}}, \\
& (\triangle+\mu-\gamma) P^{1}+(\delta+\beta-\tau) P^{0}=-2^{-1 / 2} i \mu \bar{Q}^{0^{\prime}}, \\
& (D+\bar{\epsilon}-\bar{\rho}) \bar{Q}^{0^{\prime}}+(\delta+\bar{\pi}-\bar{\alpha}) \bar{Q}^{1^{\prime}}=-2^{-1 / 2} i \mu P^{1}, \\
& (\triangle+\bar{\mu}-\bar{\gamma}) \bar{Q}^{1^{\prime}}+(\bar{\delta}+\bar{\beta}-\bar{\tau}) \bar{Q}^{0^{\prime}}=2^{-1 / 2} i \mu P^{0} .
\end{aligned}
$$


The null tetrad for the Kerr-Newman black hole can be taken as

$$
\begin{aligned}
l^{\mu} & =\left(\frac{r^{2}+a^{2}}{\Delta}, 1,0, \frac{a}{\Delta}\right), \\
n^{\mu} & =\frac{1}{2 \rho \bar{\rho}}\left(\left(r^{2}+a^{2}\right),-\Delta, 0, a\right), \\
m^{\mu} & =\frac{1}{\sqrt{2} \bar{\rho}}\left(i a \sin \theta, 0,1, \frac{i}{\sin \theta}\right),
\end{aligned}
$$

with

$$
\begin{aligned}
& \rho=r-i a \cos \theta, \\
& \Delta=r^{2}-2 M r+a^{2}+Q^{2}=\left(r-r_{+}\right)\left(r-r_{-}\right),
\end{aligned}
$$

where $M, Q$ and $a$ represent the mass, charge and angular momentum per unit mass of the Kerr-Newman black hole respectively, and $r_{ \pm}=M \pm \sqrt{M^{2}-a^{2}-Q^{2}}$. As $Q \rightarrow 0$ the KerrNewman metric reduces to the rotating Kerr metric, and as $a \rightarrow 0$ it reduces to the charged Reissner-Nordström metric. Then the wave functions can be taken as

$$
\begin{aligned}
P^{0} & =\frac{1}{\rho} \mathbb{R}_{-1 / 2}(r) S_{-1 / 2}(\theta) e^{-i(\omega t-m \varphi)}, \\
P^{1} & =\mathbb{R}_{+1 / 2}(r) S_{+1 / 2}(\theta) e^{-i(\omega t-m \varphi)}, \\
\bar{Q}^{1^{\prime}} & =\mathbb{R}_{+1 / 2}(r) S_{-1 / 2}(\theta) e^{-i(\omega t-m \varphi)}, \\
\bar{Q}^{0^{\prime}} & =-\frac{1}{\bar{\rho}} \mathbb{R}_{-1 / 2}(r) S_{+1 / 2}(\theta) e^{-i(\omega t-m \varphi)},
\end{aligned}
$$

where $\omega$ and $m$ are the energy and angular momentum of the Dirac particle. After the tedious calculation Eq. (2.2) can be simplified as

$$
\begin{aligned}
& \sqrt{\Delta} \mathcal{D}_{0} \mathbb{R}_{-1 / 2}=(\lambda+i \mu r) \sqrt{\Delta} \mathbb{R}_{+1 / 2}, \\
& \sqrt{\Delta} \mathcal{D}_{0}^{\dagger}\left(\sqrt{\Delta} \mathbb{R}_{+1 / 2}\right)=(\lambda-i \mu r) \mathbb{R}_{-1 / 2}, \\
& \mathcal{L}_{1 / 2} S_{+1 / 2}=-(\lambda-a \mu \cos \theta) S_{-1 / 2}, \\
& \mathcal{L}_{1 / 2}^{\dagger} S_{-1 / 2}=(\lambda+a \mu \cos \theta) S_{+1 / 2} .
\end{aligned}
$$

with

$$
\begin{aligned}
& \mathcal{D}_{n}=\frac{\partial}{\partial r}-\frac{i K}{\Delta}+\frac{n}{\Delta} \frac{d \Delta}{d r}, \\
& \mathcal{D}_{n}^{\dagger}=\frac{\partial}{\partial r}+\frac{i K}{\Delta}+\frac{n}{\Delta} \frac{d \Delta}{d r}, \\
& \mathcal{L}_{n}=\frac{\partial}{\partial \theta}-a \omega \sin \theta+\frac{m}{\sin \theta}+n \cot \theta, \\
& \mathcal{L}_{n}^{\dagger}=\frac{\partial}{\partial \theta}+a \omega \sin \theta-\frac{m}{\sin \theta}+n \cot \theta, \\
& K=\left(r^{2}+a^{2}\right) \omega-m a .
\end{aligned}
$$


We can eliminate $S_{+1 / 2}$ (or $\left.S_{-1 / 2}\right)$ from Eqs. (2.8) and (2.9). Defining $u=\cos \theta$, we find that the angular equation can be expressed as

$$
\frac{d}{d u}\left[\left(1-u^{2}\right) \frac{d S_{s}}{d u}\right]+\left[(a \omega u)^{2}-2 a \omega s u+s+A_{l m}-\frac{(m+s u)^{2}}{1-u^{2}}\right] S_{s}=0,
$$

where $A_{l m}$ is the angular separation constant. It is interesting to note that the angular equation (2.11) is the same as in the Kerr case.

We will focus our attention on the massless Dirac field in this paper. Therefore, we can eliminate $\mathbb{R}_{-1 / 2}$ (or $\sqrt{\Delta} \mathbb{R}_{+1 / 2}$ ) from Eqs. (2.6) and (2.7) to obtain a radial decoupled Dirac equation for $\sqrt{\Delta} \mathbb{R}_{+1 / 2}$ (or $\mathbb{R}_{-1 / 2}$ ). Then, introducing an usual tortoise coordinate

$$
d r_{*}=\frac{r^{2}+a^{2}}{\Delta} d r
$$

and resolving the equation in the form

$$
\mathbb{R}_{s}=\frac{\Delta^{-s / 2}}{\sqrt{r^{2}+a^{2}}} \Psi_{s}
$$

we obtain the wave equation

$$
\frac{d^{2} \Psi_{s}}{d r_{*}^{2}}+\left(\omega^{2}-V_{s}\right) \Psi_{s}=0
$$

with

$$
V_{s}=-\frac{d Z}{d r_{*}}+Z^{2}+\frac{\Delta}{\left(r^{2}+a^{2}\right)^{2}}\left[\frac{2 m a \omega\left(r^{2}+a^{2}\right)-m^{2} a^{2}}{\Delta}+\frac{i s K}{\Delta} \frac{d \Delta}{d r}-4 i s \omega r+\lambda^{2}\right],
$$

where $Z=-\frac{s}{2\left(r^{2}+a^{2}\right)} \frac{d \Delta}{d r}-\frac{r \Delta}{\left(r^{2}+a^{2}\right)^{2}}$ and $\lambda^{2}=A_{l m}-2 m a \omega+(a \omega)^{2}$. We will see that we can easily work out the Dirac quasinormal frequencies of the Kerr-Newman black hole from Eqs. (2.11) and (2.14) using the continued fraction approach.

\section{ANGULAR AND RADIAL CONTINUED FRACTION EQUATIONS}

For the Kerr-Newman black hole, we have to solve Eq. (2.11) numerically following Leaver 38]. Boundary conditions for Eq. (2.11) are that $S_{s}$ is regular at the regular singular points $u= \pm 1$ where the indices are given by $\pm(m+s) / 2$ at $u=1$ and $\pm(m-s) / 2$ at $u=-1$. So a solution to Eq. (2.11) may be expressed as

$$
S_{s}(u)=e^{a \omega u}(1+u)^{k_{1}}(1-u)^{k_{2}} \sum_{n=0}^{\infty} a_{n}^{\theta}(1+u)^{n}
$$

where $k_{1}=|m-s| / 2, k_{2}=|m+s| / 2$, and the superscript $\theta$ denotes the association with the angular equation. The series coefficients are related by a three term recurrence relation and 
the boundary condition at $u=+1$ is satisfied only by its minimal solution sequence. The three-term recurrence relation can be written as

$$
\begin{aligned}
& \alpha_{0}^{\theta} a_{1}^{\theta}+\beta_{0}^{\theta} a_{0}^{\theta}=0, \\
& \alpha_{n}^{\theta} a_{n+1}^{\theta}+\beta_{n}^{\theta} a_{n}^{\theta}+\gamma_{n}^{\theta} a_{n-1}^{\theta}=0, \quad(n \geq 1),
\end{aligned}
$$

where

$$
\begin{aligned}
\alpha_{n}^{\theta}= & -2(n+1)\left(n+2 k_{1}+1\right), \\
\beta_{n}^{\theta}= & n(n-1)+2 n\left(k_{1}+k_{2}+1-2 a \omega\right)-2 a \omega\left(2 k_{1}+s+1\right) \\
& +\left(k_{1}+k_{2}\right)\left(k_{1}+k_{2}+1\right)-\left[a^{2} \omega^{2}+s(s+1)+A_{l m}\right], \\
\gamma_{n}^{\theta}= & 2 a \omega\left(n+k_{1}+k_{2}+s\right) .
\end{aligned}
$$

We will obtain the minimal solution if the angular separation constant $A_{l m}$ is a root of the continued fraction equation

$$
0=\beta_{0}^{\theta}-\frac{\alpha_{0}^{\theta} \gamma_{1}^{\theta}}{\beta_{1}^{\theta}-} \frac{\alpha_{1}^{\theta} \gamma_{2}^{\theta}}{\beta_{2}^{\theta}-} \frac{\alpha_{2}^{\theta} \gamma_{3}^{\theta}}{\beta_{3}^{\theta}-} \frac{\alpha_{3}^{\theta} \gamma_{4}^{\theta}}{\beta_{4}^{\theta}-} \cdots
$$

The QNMs of the Kerr-Newman black hole are defined to be the modes with purely ingoing waves at the event horizon and purely outgoing waves at infinity [1]. Then, the boundary conditions on wave function $\Psi_{s}$ at the horizon $\left(r=r_{+}\right)$and infinity $(r \rightarrow+\infty)$ can be expressed as

$$
\Psi_{s} \sim \begin{cases}\left(r-r_{+}\right)^{-\frac{s}{2}-i \sigma_{+}} & r \rightarrow r_{+} \\ r^{-s+i \omega} e^{i \omega r} & r \rightarrow+\infty\end{cases}
$$

where $\sigma_{ \pm}=\frac{1}{r_{+}-r_{-}}\left[\left(r_{ \pm}^{2}+a^{2}\right) \omega-m a\right]$.

A solution to Eq. (2.14) which satisfies the desired behavior at the boundary can be written in the form

$$
\Psi_{s}=\sqrt{r^{2}+a^{2}}\left(r-r_{+}\right)^{-\frac{s}{2}-i \sigma_{+}}\left(r-r_{-}\right)^{-1-\frac{s}{2}+2 i \omega+i \sigma_{-}} e^{i \omega\left(r-r_{-}\right)} \sum_{n=0}^{\infty} a_{n}\left(\frac{r-r_{+}}{r-r_{-}}\right)^{n} .
$$

If we take $r_{+}+r_{-}=1$ and $b=r_{+}-r_{-}$, the sequence of the expansion coefficients $\left\{a_{n}: n=\right.$ $1,2, \ldots\}$ is determined by a three-term recurrence relation staring with $a_{0}=1$ :

$$
\begin{aligned}
& \alpha_{0} a_{1}+\beta_{0} a_{0}=0, \\
& \alpha_{n} a_{n+1}+\beta_{n} a_{n}+\gamma_{n} a_{n-1}=0, \quad(n=1,2, \ldots) .
\end{aligned}
$$

The recurrence coefficient $\alpha_{n}, \beta_{n}$ and $\gamma_{n}$ are given in terms of $n$ and the black hole parameters by

$$
\begin{aligned}
& \alpha_{n}=n^{2}+\left(C_{0}+1\right) n+C_{0}, \\
& \beta_{n}=-2 n^{2}+\left(C_{1}+2\right) n+C_{3}, \\
& \gamma_{n}=n^{2}+\left(C_{2}-3\right) n+C_{4}-C_{2}+2,
\end{aligned}
$$


and the intermediate constants $C_{n}$ are defined by

$$
\begin{aligned}
C_{0}= & 1-s-i \omega-\frac{2 i}{b}\left[\left(\frac{r_{+}^{2}+r_{-}^{2}}{2}+a^{2}\right) \omega-m a\right], \\
C_{1}= & -4+2 i \omega(2+b)+\frac{4 i}{b}\left[\left(\frac{r_{+}^{2}+r_{-}^{2}}{2}+a^{2}\right) \omega-m a\right], \\
C_{2}= & s+3-3 i \omega-\frac{2 i}{b}\left[\left(\frac{r_{+}^{2}+r_{-}^{2}}{2}+a^{2}\right) \omega-m a\right], \\
C_{3}= & \omega^{2}\left(4+2 b-4 r_{+} r_{-}+3 a^{2}\right)-2 m a \omega-s-1+(2+b) i \omega-A_{l m} \\
& +\frac{2(2 \omega+i)}{b}\left[\left(\frac{r_{+}^{2}+r_{-}^{2}}{2}+a^{2}\right) \omega-m a\right], \\
C_{4}= & s+1-2 \omega^{2}-(2 s+3) i \omega-\frac{2(2 \omega+i)}{b}\left[\left(\frac{r_{+}^{2}+r_{-}^{2}}{2}+a^{2}\right) \omega-m a\right] .
\end{aligned}
$$

It is interesting to note that the three-term recursion relation is obtained form the wave equation (2.14) directly. The calculation is simpler than other cases. For example, the coefficients of the expansion for the electromagnetic and gravitational fields in the ReissnerNordström black hole are determined by a four-term recursion relation which should be reduced to a three-term relation using a Gaussian eliminated step (see [40] for details). Our

results are also more concise than that for the electromagnetic and gravitational perturbations in the Kerr-Newman black-hole spacetime obtained by Berti and Kokkotas [25].

The series (3.7) converges and the $r=+\infty$ boundary condition (3.6) is satisfied if, for a given $s, a, m$ and $A_{l m}$, the frequency $\omega$ is a root of the continued fraction equation

$$
\left[\beta_{n}-\frac{\alpha_{n-1} \gamma_{n}}{\beta_{n-1}-} \frac{\alpha_{n-2} \gamma_{n-1}}{\beta_{n-2}-} \ldots \frac{\alpha_{0} \gamma_{1}}{\beta_{0}}\right]=\left[\frac{\alpha_{n} \gamma_{n+1}}{\beta_{n+1}-} \frac{\alpha_{n+1} \gamma_{n+2}}{\beta_{n+2}-} \frac{\alpha_{n+2} \gamma_{n+3}}{\beta_{n+3}-} \ldots\right], \quad(n=1,2 \ldots) .
$$

The solution to the radial equation (3.11) is strictly bounded up with the determination of the eigenvalue $A_{l m}$ of the angular equation (3.5), which appears explicitly in the coefficients of the recurrence relation associated with the radial equation. We could attempt to solve for both $\omega$ and $A_{l m}$ simultaneously, but this would be a time-consuming procedure. Instead, we first fix the value of $a, l, m$ and $\omega$ and find the angular separation constant $A_{l m}$ by looking for the zero of the angular continued fraction (3.5). Then, we use the corresponding eigenvalue to look for the zeros of the radial continued fraction. The $n$-th quasinormal frequency is (numerically) the most stable root of the $n$-th inversion of the continued fraction relation (3.11).

\section{NUMERICAL RESULTS}

In this section we present the numerical results obtained by using the numerical procedure just outlined in the previous section. The results will be organized into three subsections: Dirac QNMs of the Reissner-Nordström black hole, Dirac QNMs of the Kerr black hole and Dirac QNMs of the Kerr-Newman black hole. 
TABLE I: Dirac quasinormal frequencies of the Reissner-Nordström black hole for $\lambda=1$.

\begin{tabular}{c|c|c|c|c}
\hline \hline$Q$ & $\omega(n=0)$ & $\omega(n=1)$ & $\omega(n=2)$ & $\omega(n=3)$ \\
\hline 0.00 & $0.365926-0.193965 \mathrm{i}$ & $0.295644-0.633857 \mathrm{i}$ & $0.240740-1.12845 \mathrm{i}$ & $0.208512-1.63397 \mathrm{i}$ \\
0.05 & $0.366591-0.194066 \mathrm{i}$ & $0.296432-0.634048 \mathrm{i}$ & $0.241590-1.12866 \mathrm{i}$ & $0.209400-1.63420 \mathrm{i}$ \\
0.10 & $0.368615-0.194366 \mathrm{i}$ & $0.298836-0.634601 \mathrm{i}$ & $0.244190-1.12923 \mathrm{i}$ & $0.212121-1.63480 \mathrm{i}$ \\
0.15 & $0.372096-0.194851 \mathrm{i}$ & $0.302988-0.635442 \mathrm{i}$ & $0.248696-1.13001 \mathrm{i}$ & $0.216843-1.63555 \mathrm{i}$ \\
0.20 & $0.377208-0.195488 \mathrm{i}$ & $0.309132-0.636419 \mathrm{i}$ & $0.255389-1.13068 \mathrm{i}$ & $0.223871-1.63594 \mathrm{i}$ \\
0.25 & $0.384241-0.196213 \mathrm{i}$ & $0.317661-0.637242 \mathrm{i}$ & $0.264718-1.13064 \mathrm{i}$ & $0.233661-1.63507 \mathrm{i}$ \\
0.30 & $0.393651-0.196891 \mathrm{i}$ & $0.329196-0.637331 \mathrm{i}$ & $0.277339-1.12871 \mathrm{i}$ & $0.246805-1.63118 \mathrm{i}$ \\
0.35 & $0.406184-0.197220 \mathrm{i}$ & $0.344692-0.635457 \mathrm{i}$ & $0.294100-1.12248 \mathrm{i}$ & $0.263748-1.62065 \mathrm{i}$ \\
0.40 & $0.423108-0.196443 \mathrm{i}$ & $0.365519-0.628639 \mathrm{i}$ & $0.315375-1.10611 \mathrm{i}$ & $0.282749-1.59477 \mathrm{i}$ \\
0.45 & $0.446635-0.192202 \mathrm{i}$ & $0.391957-0.607831 \mathrm{i}$ & $0.333021-1.06224 \mathrm{i}$ & $0.277400-1.52872 \mathrm{i}$ \\
\hline \hline
\end{tabular}

\section{A. Dirac QNMs of the Reissner-Nordström black hole}

As the angular momentum per unit mass tends to zero $(a \rightarrow 0)$ the Kerr-Newman metric reduces to the Reissner-Nordström metric. In this limit the angular separation constant $A_{l m}=$

$\lambda^{2}=\left(l+\frac{1}{2}\right)^{2}[36]$ ( $l$ is the quantum number characterizing the angular distribution). Thus, the QNMs in this static spacetime can be obtained easily. The Dirac quasinormal frequencies of the Reissner-Nordström black hole for $n=0,1,2,3$ and $\lambda=1$ are listed in the table $I$ and that for $n=0,1,2,3$ and $\lambda=2$ are listed in the table $I I$. From the tables we find that both the real and imaginary parts of the quasinormal frequencies decrease as the overtone number increases for the fixed charge and angular quantum number (i.e., $Q=$ const. and $l=$ const.). We also find that both the real and imaginary parts of the quasinormal frequencies increases as the angular quantum number increases (except for the cases $Q=0.45$ and $n=0$ ) for the fixed charge and overtone number.

\section{B. Dirac QNMs of the Kerr black hole}

The Dirac quasinormal frequencies of the Kerr black hole for $n=0,1,2,3$ and $\lambda=1$ are listed in the table $I I I$ and that for $n=0,1,2,3$ and $\lambda=2$ are listed in the table $I V$. We find that both the real and imaginary parts of the quasinormal frequencies decrease as the overtone number increases for the fixed angular momentum per unit mass and angular quantum number. We also find that both the real and imaginary parts of the quasinormal frequencies increases as the angular quantum number increases (except for the cases $a=0.4,0.45$ and $n=0$ ) for 
TABLE II: Dirac quasinormal frequencies of the Reissner-Nordström black hole for $\lambda=2$.

\begin{tabular}{c|c|c|c|c}
\hline \hline$Q$ & $\omega(n=0)$ & $\omega(n=1)$ & $\omega(n=2)$ & $\omega(n=3)$ \\
\hline 0.00 & $0.760074-0.192810 \mathrm{i}$ & $0.711666-0.595995 \mathrm{i}$ & $0.638523-1.03691 \mathrm{i}$ & $0.569867-1.51490 \mathrm{i}$ \\
0.05 & $0.761372-0.192916 \mathrm{i}$ & $0.713058-0.595276 \mathrm{i}$ & $0.640042-1.03728 \mathrm{i}$ & $0.571477-1.51529 \mathrm{i}$ \\
0.10 & $0.765327-0.193230 \mathrm{i}$ & $0.717299-0.596107 \mathrm{i}$ & $0.644677-1.03836 \mathrm{i}$ & $0.576395-1.51643 \mathrm{i}$ \\
0.15 & $0.772119-0.193738 \mathrm{i}$ & $0.724597-0.597440 \mathrm{i}$ & $0.652672-1.04004 \mathrm{i}$ & $0.584893-1.51813 \mathrm{i}$ \\
0.20 & $0.782084-0.194414 \mathrm{i}$ & $0.735335-0.599172 \mathrm{i}$ & $0.664476-1.04210 \mathrm{i}$ & $0.597473-1.52001 \mathrm{i}$ \\
0.25 & $0.795772-0.195199 \mathrm{i}$ & $0.750138-0.601099 \mathrm{i}$ & $0.680818-1.04411 \mathrm{i}$ & $0.614939-1.52137 \mathrm{i}$ \\
0.30 & $0.814058-0.195973 \mathrm{i}$ & $0.770003-0.602799 \mathrm{i}$ & $0.702848-1.04523 \mathrm{i}$ & $0.638525-1.52082 \mathrm{i}$ \\
0.35 & $0.838381-0.196464 \mathrm{i}$ & $0.796544-0.603349 \mathrm{i}$ & $0.732368-1.04363 \mathrm{i}$ & $0.670048-1.51544 \mathrm{i}$ \\
0.40 & $0.871259-0.195997 \mathrm{i}$ & $0.832478-0.600485 \mathrm{i}$ & $0.772116-1.03491 \mathrm{i}$ & $0.711672-1.49828 \mathrm{i}$ \\
0.45 & $0.918762-0.192368 \mathrm{i}$ & $0.882263-0.587382 \mathrm{i}$ & $0.824382-1.00617 \mathrm{i}$ & $0.760227-1.44942 \mathrm{i}$ \\
\hline \hline
\end{tabular}

the fixed angular momentum per unit mass and overtone number.

By comparing the results of the Reissner-Nordström and Kerr black holes, it is interesting to note that the real part of the quasinormal frequencies of the Reissner-Nordström black hole is greater than that of the Kerr black hole, but the imaginary part of the quasinormal frequencies of the Reissner-Nordström black hole is less than that of the Kerr black hole if we take the same values of the charge and the angular momentum per unit mass. That is to say, the decay of the Dirac fields in the charged black-hole spacetime is faster than that in the rotating black-hole spacetime if the values of the charge and angular momentum per unit mass are the same.

\section{Dirac QNMs of the Kerr-Newman black hole}

The Dirac quasinormal frequencies of the Kerr-Newman black hole for $n=0,1,2$ and $\lambda=1$ are shown by figure 1 and those for $n=5,6,7$ and $\lambda=2$ are shown by figure 2 . The left columns in the Figs. 1 and 2 describe the behavior of quasinormal frequencies in the complex $\omega$ plane which show that the frequencies generally move counterclockwise as the charge (the thick curves) or the angular momentum per unit mass (the thin curves) increases. They get a spiral-like shape, moving out of the Schwarzschild ( $Q=0$ and $a=0$ ) or Reissner-Nordström $(0<Q<0.5$ and $a=0)$ values and "looping in" towards some limiting frequencies as the charge and angular momentum per unit mass tend to the extremal value $\sqrt{a^{2}+Q^{2}}=1 / 2$. For a given $\lambda$, we observe that the number of spirals increases as the overtone number increases. However, for a given overtone number $n$, the increasing $\lambda$ has the effect of "unwinding" the 
TABLE III: Dirac quasinormal frequencies of the Kerr black hole for $\lambda=1$.

\begin{tabular}{c|c|c|c|c}
\hline \hline$a$ & $\omega(n=0)$ & $\omega(n=1)$ & $\omega(n=2)$ & $\omega(n=3)$ \\
\hline 0.00 & $0.365926-0.193965 \mathrm{i}$ & $0.295644-0.633857 \mathrm{i}$ & $0.240740-1.12845 \mathrm{i}$ & $0.208512-1.63397 \mathrm{i}$ \\
0.05 & $0.366218-0.193805 \mathrm{i}$ & $0.296135-0.633141 \mathrm{i}$ & $0.241239-1.12700 \mathrm{i}$ & $0.208875-1.63180 \mathrm{i}$ \\
0.10 & $0.367099-0.193316 \mathrm{i}$ & $0.297605-0.630951 \mathrm{i}$ & $0.242707-1.12257 \mathrm{i}$ & $0.209884-1.62514 \mathrm{i}$ \\
0.15 & $0.368589-0.192461 \mathrm{i}$ & $0.300054-0.627144 \mathrm{i}$ & $0.245045-1.11486 \mathrm{i}$ & $0.211268-1.61355 \mathrm{i}$ \\
0.20 & $0.370717-0.191175 \mathrm{i}$ & $0.303460-0.621458 \mathrm{i}$ & $0.248029-1.10334 \mathrm{i}$ & $0.212433-1.59615 \mathrm{i}$ \\
0.25 & $0.373525-0.189350 \mathrm{i}$ & $0.307751-0.613449 \mathrm{i}$ & $0.251166-1.08710 \mathrm{i}$ & $0.212101-1.57147 \mathrm{i}$ \\
0.30 & $0.377060-0.186806 \mathrm{i}$ & $0.312708-0.602389 \mathrm{i}$ & $0.253306-1.06460 \mathrm{i}$ & $0.207277-1.53691 \mathrm{i}$ \\
0.35 & $0.381352-0.183245 \mathrm{i}$ & $0.317679-0.587057 \mathrm{i}$ & $0.251404-1.03327 \mathrm{i}$ & $0.189183-1.48777 \mathrm{i}$ \\
0.40 & $0.386329-0.178141 \mathrm{i}$ & $0.320563-0.565425 \mathrm{i}$ & $0.234871-0.98938 \mathrm{i}$ & $0.106512-1.41213 \mathrm{i}$ \\
0.45 & $0.391413-0.170595 \mathrm{i}$ & $0.313069-0.536419 \mathrm{i}$ & $0.178522-0.99702 \mathrm{i}$ & $0.067565-1.44066 \mathrm{i}$ \\
\hline \hline
\end{tabular}

TABLE IV: Dirac quasinormal frequencies of the Kerr black hole for $\lambda=2$.

\begin{tabular}{c|c|c|c|c}
\hline \hline$a$ & $\omega(n=0)$ & $\omega(n=1)$ & $\omega(n=2)$ & $\omega(n=3)$ \\
\hline 0.00 & $0.760074-0.192810 \mathrm{i}$ & $0.711666-0.594995 \mathrm{i}$ & $0.638523-1.03691 \mathrm{i}$ & $0.569867-1.51490 \mathrm{i}$ \\
0.05 & $0.760593-0.192662 \mathrm{i}$ & $0.712351-0.594475 \mathrm{i}$ & $0.639418-1.03584 \mathrm{i}$ & $0.570876-1.51315 \mathrm{i}$ \\
0.10 & $0.762165-0.192207 \mathrm{i}$ & $0.714416-0.592884 \mathrm{i}$ & $0.642106-1.03257 \mathrm{i}$ & $0.573887-1.50779 \mathrm{i}$ \\
0.15 & $0.764829-0.191416 \mathrm{i}$ & $0.717897-0.590117 \mathrm{i}$ & $0.646595-1.02688 \mathrm{i}$ & $0.578836-1.49851 \mathrm{i}$ \\
0.20 & $0.768656-0.190231 \mathrm{i}$ & $0.722849-0.585981 \mathrm{i}$ & $0.652877-1.01841 \mathrm{i}$ & $0.585563-1.48468 \mathrm{i}$ \\
0.25 & $0.773753-0.188558 \mathrm{i}$ & $0.729342-0.580157 \mathrm{i}$ & $0.660882-1.00652 \mathrm{i}$ & $0.593690-1.46530 \mathrm{i}$ \\
0.30 & $0.780274-0.186246 \mathrm{i}$ & $0.737432-0.572132 \mathrm{i}$ & $0.670362-0.99020 \mathrm{i}$ & $0.602309-1.43876 \mathrm{i}$ \\
0.35 & $0.788430-0.183045 \mathrm{i}$ & $0.747081-0.561063 \mathrm{i}$ & $0.680552-0.96784 \mathrm{i}$ & $0.609060-1.40255 \mathrm{i}$ \\
0.40 & $0.798494-0.178518 \mathrm{i}$ & $0.757886-0.545547 \mathrm{i}$ & $0.689090-0.93700 \mathrm{i}$ & $0.607082-1.35381 \mathrm{i}$ \\
0.45 & $0.810750-0.171857 \mathrm{i}$ & $0.768079-0.523427 \mathrm{i}$ & $0.688731-0.89635 \mathrm{i}$ & $0.580342-1.30276 \mathrm{i}$ \\
\hline \hline
\end{tabular}

spirals, as we see in the two figures that the spiral begins at $n=2$ for $\lambda=1$ but starts at $n=6$ for $\lambda=2$.

The second and last columns in the Figs. 1 and 2 illustrate that the real and imaginary parts of the quasinormal frequencies are the functions of the angular momentum per unit mass $a$. We know from these figures that both the real and imaginary parts of the frequencies are oscillatory functions of the angular momentum per unit mass. The oscillation starts earlier 

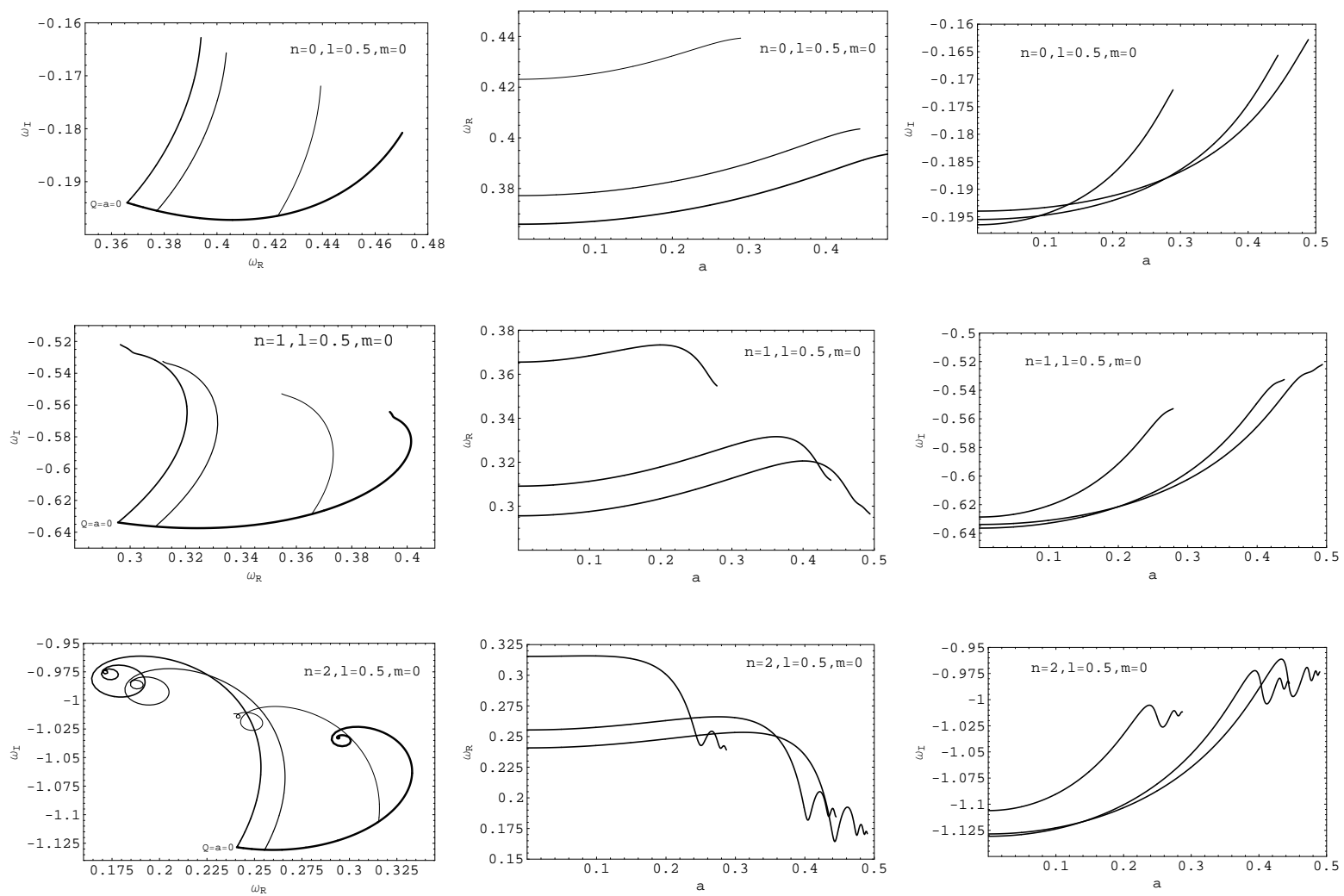

FIG. 1: The left three panels show trajectories in the complex $\omega$ plane of the first three Dirac QNMs of the Kerr-Newman black hole for $l=1 / 2$ and $m=0$. In each panel, the thick curve corresponds to the Reissner-Nordström black hole $(\mathrm{a}=0)$ which is drawn by increasing $Q$ from zero to the extremal limit and the three thin curves are obtained by increasing $a$ from zero to the extremal limit for fixed values of the charge $Q=0,0.2,0.4$ (left to right). These curves show that the frequencies generally move counterclockwise as $a$ (or $Q$ ) increases and the number of spirals increases as the overtone number increases. The other panels draw $\operatorname{Re}(\omega)$ and $\operatorname{Im}(\omega)$ of the quasinormal frequencies versus $a$ in which the curves from bottom to top refer to $Q=0,0.2,0.4$. These panels tell us that, for $n \geq 2$, both the real and imaginary parts are oscillatory functions of $a$ and the oscillations become faster as the overtone number increases.

and earlier as the overtone number $n$ grows for a fixed $\lambda$, but it begins later and later as the angular quantum number $a$ increases for a fixed $n$. We also learn that the oscillation starts earlier and earlier as the charge $Q$ increases for fixed $n$ and $\lambda$. Meanwhile, the oscillation becomes faster as the overtone number increases for a given $\lambda$, but it becomes slower as $\lambda$ increases for a given overtone number $n$. 

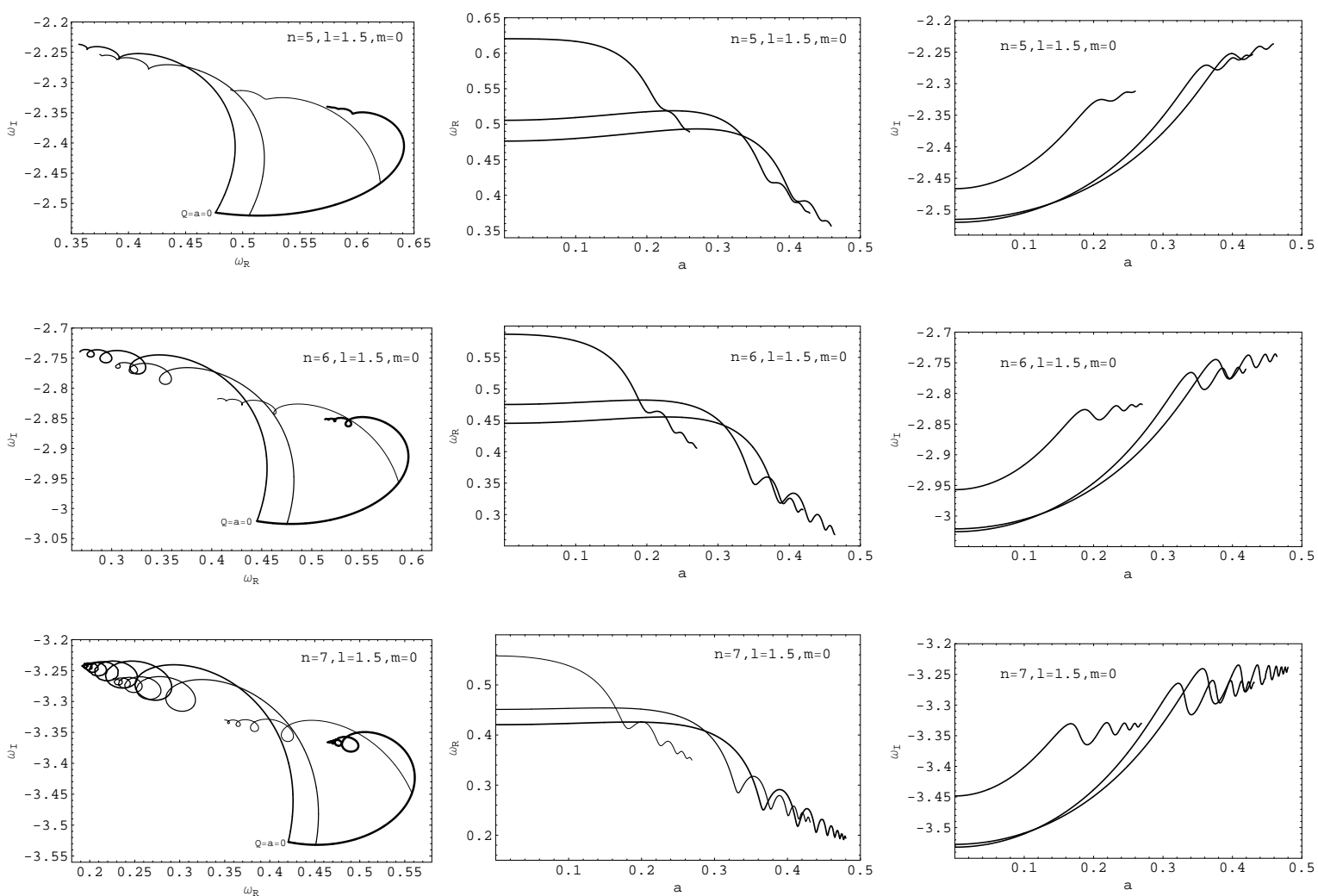

FIG. 2: The left three panels describe the behavior of the Dirac quasinormal frequencies of the Kerr-Newman black hole for $n=5,6,7, l=3 / 2$ and $m=0$ in the complex $\omega$ plane. In each panel, the thick curve is drawn by increasing $Q$ from zero to the extremal limit for $a=0$ and the three thin curves are obtained by increasing $a$ from zero to the extremal limit for fixed values of the charge $Q=0,0.2,0.4$ (left to right). The other panels draw $\operatorname{Re}(\omega)$ and $\operatorname{Im}(\omega)$ of the quasinormal frequencies versus $a$ in which the curves from bottom to top refer to $Q=0,0.2,0.4$. These panels tell us that, for $n \geq 5$, both the real and imaginary parts are oscillatory functions of $a$ and the oscillations begin earlier as the charge increases.

\section{SUMMARY}

The wave equations for the Dirac fields in the Kerr-Newman black-hole spacetime are obtained by means of the Newman-Penrose formalism. The expansion coefficients of the wave equation which satisfy appropriate boundary conditions are directly determined by a threeterm recurrence relation. Then, the Dirac quasinormal frequencies of the Kerr-Newman black hole are evaluated using continued fraction approach and the results are presented by tables and figures. By comparing the results of the Reissner-Nordström and Kerr black holes, it is interesting to note that the real part of the quasinormal frequencies of the Reissner-Nordström black hole is greater than that of the Kerr black hole, but the imaginary part is less than that 
of the Kerr black hole if we take the same values of the charge and the angular momentum per unit mass. That is to say, the decay of the Dirac fields in the charged black-hole spacetime is faster than that in the rotating black-hole spacetime if the values of the charge and angular momentum per unit mass are the same. From figures we find that the frequencies in the complex $\omega$ plane generally move counterclockwise as the charge or the angular momentum per unit mass increases. They get a spiral-like shape, moving out of their Schwarzschild or Reissner-Nordström values and "looping in" towards some limiting frequencies as the charge and angular momentum per unit mass tend to their extremal values. For a given angular quantum number $\lambda$, we observe that the number of spirals increases as the overtone number increases. However, for a given overtone number, the increasing $\lambda$ has the effect of "unwinding" the spirals. We also find that the real and imaginary parts of the quasinormal frequencies are oscillatory functions of the angular momentum per unit mass, and the oscillating behavior starts earlier and earlier as the overtone number grows for a fixed $\lambda$ (or as the charge $Q$ increases for fixed $n$ and $\lambda$ ), but it begins later and later as $\lambda$ increases for a fixed overtone number.

\section{Acknowledgments}

This work was supported by the National Natural Science Foundation of China under Grant No. 10473004; the FANEDD under Grant No. 200317; and the SRFDP under Grant No. 20040542003; the Hunan Provincial Natural Science Foundation of China under Grant No. 04JJ3019; and the National Basic Research program of China under Grant No. 2003CB716300.

[1] S. Chandrasekhar and S. Detweiler, Proc. R. Soc. Lond. A 344, 441 (1975).

[2] V. P. Frolov and I.. D. Novikov, Black hole physics: basic concepts and new developments (Kluwer Academic, Dordrecht, 1998).

[3] S. Hod, Phys. Rev. Lett. 81, 4293 (1998).

[4] O. Dreyer, Phys. Rev. Lett. 90, 081301 (2003).

[5] J. Baez, in Matters of gravity. ed. J. Pullin, p. 12(Springer, 2003), gr-qc/0303027.

[6] G. Kunstatter, gr-qc/0212014 L. Motl, gr-qc/0212096 A. Corichi, gr-qc/0212126 L. Motl and A. Neitzke, hep-th/03301173; A. Maassen van den Brink, gr-qc/0303095.

[7] J. Maldacena, Adv. Theor. Math. Phys. 2, 231 (1998).

[8] E. Witten, Adv. Theor. Math. Phys. 2, 253 (1998).

[9] S. Kalyana Rama and B. Sathiapalan, Mod. Phys. Lett. A 14, 2635 (1999).

[10] J. S. F. Chan and R. B. Mann, Phys. Rev. D 55, 7546 (1997); 59, 064025 (1999).

[11] G. T. Horowitz and V. E. Hubeny, Phys. Rev. D 62, 024027 (2000). 
[12] V. Cardoso and J. P. S. Lemos, Phys. Rev. D 64, 084017 (2001).

[13] V. Cardoso, R. Konoplya and J. P. S. Lemos, gr-qc/0305037.

[14] R. A. Konoplya, Phys. Rev. D 66, 044009 (2002).

[15] B. Wang, C. Y. Lin and E. Abdalla, Phys. Lett. B 481, 79 (2000).

[16] B. Wang, C. Y. Lin and C. Molona, hep-th/0407024.

[17] I. G. Moss and J. P. Norman, Class. Quant. Grav. 19, 2323 (2002).

[18] D. Birmingham, I. Sachs and S. N. Solodukhin, Phys. Rev. Lett. 88, 151301 (2002).

[19] V. Cardoso and J. P. S. Lemos, Phys. Rev. D 63, 124015 (2001).

[20] Songbai Chen and Jiliang Jing, Class. Quant. Grav. 22, 533 (2005).

[21] Songbai Chen and Jiliang Jing, Class. Quant. Grav. 22, 1129 (2005).

[22] Songbai Chen and Jiliang Jing, Class. Quant. Grav. 22, 2159 (2005).

[23] A. O. Starinets, Phys. Rev. D 66, 124013 (2002).

[24] R. Aros, C. Martinez, R. Troncoso and J. Zanelli, Phys. Rev. D 67, 044014 (2003).

[25] E. Berti and K. D. Kokkotas, Phys. Rev. D 71, 124008 (2005).

[26] M. Giammatteo and Ian G. Moss, Class. Quant. Grav. 22, 1803 (2005).

[27] R. A. Konoplya, Phys. Rev. D 66, 084007 (2002).

[28] A. Nunez and A. O. Starinets, Phys. Rev. D 67, 124013 (2002).

[29] Y. Kurita and M. A. Sakagami, Phys. Rev. D 67, 024003 (2003).

[30] H. T. Cho, Phys. Rev. D 68, 024003 (2003).

[31] Jia-Feng Chang and You-Gen Shen, Nucl. Phys. B 712, 347 (2005).

[32] Fu-Wen Shu and You-Gen Shen, gr-qc/0501098.

[33] A. Zhidenko, Class. Quant. Grav. 21, 273 (2004).

[34] Jiliang Jing, Phys. Rev. D 69, 084009 (2004).

[35] Jiliang Jing, Phys. Rev. D 70, 065004 (2004).

[36] Jiliang Jing, Phys. Rev. D 71, 124006 (2005).

[37] Jiliang Jing, Phys. Rev. D 71, 124011 (2005).

[38] E. W. Leaver, Proc. R. Soc. Lond. A 402, 285 (1985); E. W. Leaver, Phys. Rev. D 34, 384 (1986).

[39] B. Majumdar and N. Panchapakesan, Phys. Rev. D 40, 2568 (1989).

[40] E. W. Leaver, Phys. Rev. D 41, 2986 (1990).

[41] R. P. Kerr, Phys. Rev. Lett. 11, 237 (1963); E. T. Newman, E. Couch, K. Chinnapared, A. Exton, A. Prakash and R. Torrence, J. Math. Phys. (N. Y.) 6, 918 (1965).

[42] D. N. Page, Phys. Rev. D 14, 1509 (1976).

[43] E. Newman and R. Penrose, J. Math. Phys. (N. Y.) 3, 566 (1962).

[44] N. Andersson and H. Onozawa, Phys. Rev. D 54, 7470 (1996). 